\title{
Specific ion interactions with aromatic rings in aqueous solutions: Comparison of molecular dynamics simulations with a thermodynamic solute partitioning model and Raman hydration shell spectroscopy
}

Jordan C. Vincent, ${ }^{\mathrm{a}}$ Sarah M. Matt, ${ }^{\mathrm{b}}$ Blake M. Rankin, ${ }^{\mathrm{b}}$ Raffaella D'Auria, ${ }^{\mathrm{a}, \mathrm{c}}$ J. Alfredo Freites, ${ }^{\mathrm{a}}$ Dor Ben-Amotz, ${ }^{\mathrm{b}}$ Douglas J. Tobias ${ }^{\mathrm{a}, *}$

${ }^{a}$ Department of Chemistry, University of California, Irvine, California 92697-2025 USA

${ }^{\mathrm{b}}$ Department of Chemistry, Purdue University, West Lafayette, Indiana 47907 USA

${ }^{c}$ Current address: Institute for Digital Research and Education, University of California, Los Angeles 90095 USA

*Corresponding author.

Address: Department of Chemistry, 1102 Natural Sciences 2, University of California, Irvine, CA 92697-2025, USA. Tel.: +1 (949) 824 4295. Fax: +1 (949) 8249920.

E-mail address: dtobias@uci.edu (D. J. Tobias)

Keywords: Hofmeister series, specific ion effects, molecular dynamics simulations, solute partitioning model, Raman hydration shell spectroscopy 


\begin{abstract}
Specific ion interactions of $\mathrm{KF}$, and the $\mathrm{Na}^{+}$salts of $\mathrm{SO}_{4}{ }^{2-}, \mathrm{F}^{-}, \mathrm{Cl}^{-}, \mathrm{NO}_{3}{ }^{-}, \mathrm{I}^{-}$, and $\mathrm{ClO}_{4}^{-}$with benzene in aqueous solutions were investigated using molecular dynamics simulations and compared with experimental Raman multivariate curve resolution (Raman-MCR) and thermodynamic results. Good agreement is found with the hydration-shell partition coefficients of salts obtained from the thermodynamic analysis and of halogen anions obtained from the Raman-MCR spectra of benzene and pyridine. Larger discrepancies between the simulation and thermodynamic cation partitioning results point to the influence of counter-ion interaction on cation partitioning.
\end{abstract}




\section{Introduction}

In the late 1800s, Franz Hofmeister discovered that the extent to which proteins precipitated depends on the identity of the salts present in solution (1). Salt ions could be consistently ranked according to their effect on protein solubility in an order that is now referred to as the Hofmeister series. A representative ranking of anions, listed in decreasing order of ability to precipitate proteins is: $\mathrm{CO}_{3}{ }^{2-}>\mathrm{SO}_{4}{ }^{2-}>\mathrm{F}^{-}>\mathrm{Cl}^{-}>\mathrm{Br}^{-} \sim \mathrm{NO}_{3}{ }^{-}>\mathrm{I}^{-}>\mathrm{ClO}_{4}{ }^{-}$(2). Ions on the left side of the series "salt out" (precipitate) proteins and those on the right "salt in" (dissolve/denature) proteins. An analogous series also exists for cations. Since its discovery, the Hofmeister series has been shown to arise in a wide variety of seemingly unrelated solution phenomena, including protein solubility, surface tension, water retention in wool, and bacterial growth, among many others $(3,4)$.

Until fairly recently it was thought that the Hofmeister series followed from the "structure making" (kosmotropic) or "structure breaking" (chaotropic) effects of ions on water structure $(3,5)$. However, more recent computational and Raman spectroscopic studies (6-8), as well as ultra-fast infrared pump-probe (9), x-ray absorption (10), and terahertz $(11,12)$ spectroscopic measurements, demonstrated that the hydrogen-bond network in water is not affected much beyond the first hydration shell of the ions. In this paper, we will use the terms kosmotrope and chaotrope, not to imply structure water making/breaking effects, but rather to refer to where the ions lie in the Hofmeister series, kosmotropes being on the salting out (left) side and chaotropes on the salting in (right) side of the series.

Numerous computational and experimental studies, reviewed recently (2,13-16), have focused on specific ion effects at the air/water interface and at protein surfaces. Many factors have been correlated with the specificity of ions at these surfaces, including ion size, 
polarizability, valency, and concentration, as well as the identity of other ions in solution. A spectroscopic and computational study recently showed that anion adsorption to the air-water interface is favored by enthalpy and opposed by entropy (17). A free energy partitioning approach suggested recently that the dominant thermodynamic driving forces for adsorption of an iodide to the air-water interface are the reduction of the size of the cavity that accompanies adsorption and a far-field electrostatic contribution corresponding to the ion interaction with the interfacial surface potential (18). Despite these many studies, the specificity and strength of the interactions between various ions and particular compounds or molecular sub-groups remain to be fully characterized. Here we focus on interactions between ions and aromatic rings, specifically, benzene and pyridine, in water, as such interactions are of both fundamental interest and potential biological relevance because of the prevalence of aromatic side chains in the hydrocarbon core of globular proteins.

Molecular dynamics (MD) simulations have played an important role in elucidating some of the mechanisms contributing to ionic specificity in aqueous interfacial processes $(13,19)$. In this study we employ MD simulations to evaluate the affinity of various ions for benzene in several aqueous salt solutions. Furthermore, we compare our simulation results with those obtained from a recent thermodynamic analysis of the partitioning of ions between the benzene hydration layer and bulk aqueous solution (20), as well as with new Raman multivariate curve resolution (Raman-MCR) vibrational hydration-shell spectroscopic measurements of the affinity of halogen anions for both benzene and pyridine.

Record and coworkers have applied thermodynamic models to interpret and predict Hofmeister ion effects on surface tension at the air/water interface $(21,22)$ and ion interactions with nonpolar and amide groups (20). The model assessed in the present work is a two-phase 
equilibrium solute partitioning model (SPM) (20), in which the ions in an aqueous salt solution are assumed to act independently as they partition between a local hydration layer of the nonelectrolyte (e.g., benzene) solute and the bulk solution. The key parameters extracted from the SPM are ion partition coefficients, $K_{p, i}=m_{i}^{\text {local }} / m_{i}^{\text {bulk }}$, where $m_{i}^{\text {local }}$ and $m_{i}^{\text {bulk }}$, are the local (i.e., vicinity of the solute) and bulk molal concentrations, respectively, of ion $i . K_{p, i}$ provides a quantitative measure of the affinity of an ion for the solute. In this paper we will compare partition coefficients computed from our MD simulations and Raman hydration-shell spectroscopic measurements with those obtained by applying the SPM to benzene solubility data (20).

\section{Methods}

\subsection{System setup and simulation protocols}

We performed MD simulations of a single benzene molecule dissolved in aqueous salt solutions containing approximately $1 \mathrm{M} \mathrm{KF}, \mathrm{NaCl}, \mathrm{Na}_{2} \mathrm{SO}_{4}, \mathrm{NaNO}_{3}, \mathrm{NaI}, \mathrm{NaClO}_{4}$, or neat water. Nonpolarizable force fields with the TIP3P water model (23) were employed in all of the simulations. For benzene, we utilized a force field developed by Jorgensen and Severance (24). The ion parameters, along with their respective references are summarized in Table 1.

Each simulation was run for $100 \mathrm{~ns}$ with a $1 \mathrm{fs}$ time step. Coordinates were saved every $1 \mathrm{ps}$, yielding 100,000 frames for analysis. The simulation box was approximately $26.3 \times 26.3 \times 26.3$ $\AA^{3}$ and contained approximately 400 water molecules, one benzene molecule, 7 cations, and 7 anions, so that the benzene concentration was $\sim 0.09 \mathrm{M}$ and the salt concentration was $\sim 1 \mathrm{M}$. Three-dimensional periodic boundary conditions were applied and long-range electrostatic

interactions beyond $8 \AA$ were treated using the smooth particle mesh Ewald method (25). The 
Berendsen thermostat with a coupling constant of 1 ps and barostat with a coupling time of 1 ps were used to maintain a temperature of $300 \mathrm{~K}$ and a pressure of 1 atm (26). Bonds to hydrogen atoms were constrained using the SHAKE algorithm (27). All of the MD simulations were performed using the Amber 8 program (28).

\subsection{Calculation of partition coefficients from MD simulations}

Mass densities of salt ions and water molecules were averaged over 9,800 frames sampled evenly over the 100,000 frames and binned using the VMD (29) VolMap tool with bin volumes of $0.2 \AA^{3}$. The density data were binned relative to the benzene molecular center-of-mass placed at the origin, with the benzene six-carbon plane parallel to the Cartesian $x-y$ plane. Interactions between benzene and the ions/solution were assumed to be cylindrically symmetric about the benzene six-fold symmetry axis. Thus, for fixed cylindrical $(r, z, \theta)$ coordinates, volumetric mass densities of salt ions and water molecules were averaged over the azimuth $(\theta)$, and subsequently projected onto a grid in a two-dimensional $(r, z)$ coordinate system.

For the purpose of calculating ion partition coefficients, a "local" region was defined around the benzene molecule as the cylindrical volume defined by $r \leq 6.5 \AA$ and $|z| \leq 5 \AA$; this region encapsulated the first hydration shell around benzene in neat water. The remaining volume of the simulation box constitutes the "bulk" region. The ion partition coefficients, $K_{p, i}$ were calculated from the ion and mass densities within the local and bulk regions using the following relation:

$$
K_{p, i}=\frac{m_{i}^{\text {local }}}{m_{i}^{\text {bulk }}}=\frac{\rho_{i}^{\text {local }}}{\rho_{i}^{\text {bulk }}} \frac{\rho_{\text {wat }}^{\text {bulk }}}{\rho_{\text {wat }}^{\text {local }}}
$$

where $m_{i}^{j}$ is the molality of ion $i$ in the $j$ th region $\left(j=\right.$ local or bulk), $\rho_{i}^{j}$ and $\rho_{w a t}^{j}$ are the corresponding ion and water mass densities, respectively. For a salt consisting of monovalent 
ions, the salt partition coefficient is defined as $K_{p}=K_{p,+}+K_{p,-}$, where $K_{p,+}$ and $K_{p,-}$ are, respectively, the cation and anion partition coefficients (20).

\subsection{Raman-MCR spectroscopy}

High signal-to-noise Raman spectra were collected using a $514.5 \mathrm{~nm}$ Ar-ion laser excitation source with approximately $15 \mathrm{~mW}$ laser power at the sample. The backscattered Raman photons were collected using a fiber optic cable and dispersed onto a CCD camera using a 300 grooves/mm grating, as previously described (30). Some of the experiments were performed with a higher resolution grating (1200 grooves/mm); however, the results were independent of resolution. Self-modeling curve resolution (SMCR) (31), which requires no assumptions regarding the spectral shape or component concentrations of the input Raman spectra, was used to obtain the solute-correlated (SC) spectra of benzene and pyridine in aqueous $\mathrm{KF}, \mathrm{NaCl}$, and NaI solutions. Vibrational features arising from the solute (benzene or pyridine) appear in the SC spectra, as well as perturbed water molecules that differ in structure from bulk water. The three component system consisting of water, salt, and benzene (or pyridine) was reduced to a two component system by varying the concentration of only one component at a given time, as previously described (8). $\mathrm{CH}$ stretching frequencies were obtained by fitting the $\mathrm{CH}$ stretching mode at $\sim 3070 \mathrm{~cm}^{-1}$ to a Gaussian function. The ion-induced frequency shifts in this work are reported as a difference in frequency between the salt solution and salt-free solution.

The following chemicals were obtained from Sigma-Aldrich: pyridine (anhydrous, 99.8\%), potassium fluoride (ACS reagent, $\geq 99.0 \%$ ), sodium iodide (ACS reagent, $\geq 99.5 \%$ ), sodium nitrate (ACS reagent), sodium perchlorate (ACS reagent, $\geq 98.0 \%$ ), sodium sulfate (anhydrous, powder, ACS reagent, $\geq 99.0 \%$ ) and sodium fluoride (BioXtra, $\geq 99 \%$ ). Benzene and sodium chloride (Analytical Reagent) were purchased from EM Science and Mallinckrodt, respectively. 
All of the above chemicals were used as received. Aqueous samples were prepared using ultrapure water (Milli-Q UF Plus, $18.2 \mathrm{~m} \Omega \cdot \mathrm{cm}$ resistance, Millipore).

\section{Results and discussion}

\subsection{Comparison of MD and SPM results}

Fig. 1a and $1 \mathrm{~b}$ show, respectively, cation and anion $K_{p}$ values obtained from the MD simulations (black bars) and the SPM thermodynamic study (red bars) (20). Due to the variability of the $\mathrm{Na}^{+} K_{p}$ values computed from the simulations, there is no discernable difference in the behavior of $\mathrm{Na}^{+}$and $\mathrm{K}^{+}$in the vicinity of benzene. The qualitative ordering of the cation $K_{p}$ values (Fig. 1a) mirrors that of the anions (Fig. 1b). In all of the salt solutions the cations have $K_{p}$ values less than 0.72 , indicating their overall propensity for solvation in the bulk solution. The cation $K_{p}$ data are, however, inconsistent with the thermodynamic study (SPM) by up to an order of magnitude discrepancy.

It is remarkable that the anion $K_{p}$ values (Figure 1b) follow the same qualitative trend as the SPM data, except in the case of iodide. The $K_{p}$ values computed from the simulations for the chaotropic anions nitrate and perchlorate are greater than 1. However, the typically strong chaotropic anion iodide has a $K_{p} \approx 0.8$, indicating a slight preference for solvation in the bulk solution. This atypical behavior of iodide may be a consequence of the nonpolarizable force field used in this study. In previous work, polarization has been shown to enhance the propensity of heavy halides for nonpolar solute surfaces (32), and it is now well established that explicit inclusion of electronic polarization in MD simulations enhances the propensity of heavy halides for the air/water interface (13).

For the most part, the anion $K_{p}$ values calculated from the MD simulations are substantially lower than those extracted from the SPM analysis (Fig. 1b). In the case of sulfate, Pegram and 
Record assumed $K_{p}=0$ (20). Although our simulations show a very high degree of exclusion of sulfate from the benzene hydration layer, this exclusion is not absolute. Moreover, we find that the strong kosmotrope fluoride also has a small $K_{p}$ value, whereas Pegram and Record find a value close to 1 , indicating nearly equal preference of fluoride for the bulk water and the benzene hydration layer. Our simulations also yield a small $K_{p}$ for chloride indicating a preference for its solvation in bulk solution, while the SPM predicts a value greater than unity, indicating an affinity for the benzene surface.

Fig. 1c shows a comparison of the calculated overall salt $K_{p}$ values from this study (black bars) with those from the SPM study (red bars) (20). The ordering of the salt $K_{p}$ values from the MD simulations follows that of the cations and anions, which is consistent with Pegram and Record, except for the case of NaI. Excepting sodium sulfate, it is remarkable that the overall salt $K_{p}$ values derived from simulations are in near quantitative agreement with those derived from the thermodynamic study (Fig. 1c). Although sodium cations and sulfate anions are highly excluded, our simulations clearly show that neither ion is completely excluded from the hydration layer of benzene.

Given the chosen parameters defining the hydration layer about benzene, and assuming that the solvent-accessible surface area of benzene is $212 \AA^{2}(20)$, we find that there are approximately $0.17 \mathrm{H}_{2} \mathrm{O} \AA^{-2}$, which is in good agreement with the $0.18 \mathrm{H}_{2} \mathrm{O} \AA^{-2}$ assumed in the SPM (20).

The agreement between the MD and SPM salt $K_{p}$ values suggests that the thermodynamic model correctly predicts the salt $K_{p}$. However, the MD results suggest that the SPM may overestimate the anion $K_{p}$ values and underestimates the cation $K_{p}$ values, as the SPM may not properly account for counter-ion interactions. Indeed, to derive ionic $K_{p}$ values from the SPM 
analysis it was necessary for Pegram and Record to assume the ions did not interact (20).

\subsection{Comparison of $M D$ and Raman-MCR results}

Fig. 2 shows experimental results obtained from an SMCR analysis of Raman spectra of solutions containing either benzene or pyridine dissolved in aqueous $\mathrm{KF}, \mathrm{NaCl}, \mathrm{NaBr}$, or $\mathrm{NaI}$ solutions (with salt molalities between 0.1 and 1). The unprocessed spectra of the benzene solutions are virtually indistinguishable from those of the corresponding solvent (because the solubility limit of benzene is $\sim 0.02 \mathrm{~m}$ ), while the higher concentration pyridine solutions show clear evidence of pyridine vibrational bands. However, in both cases SMCR analysis may be used to obtain solute-correlated spectra that clearly reveal the $\mathrm{CH}$ stretch band of the solute (33). For example, Fig. 2 compares the solute-correlated spectra (in the $\mathrm{CH}$ and $\mathrm{OH}$ stretch region) of $0.5 \mathrm{~m}$ pyridine in pure water and in $1 \mathrm{~m} \mathrm{NaI}$ (compared with the $\mathrm{OH}$ stretch band of pure water). Fig. 2a shows a close up view of the $\mathrm{CH}$ peak of pyridine which reveals a small but measureable red-shift of $\Delta v \sim 2 \mathrm{~cm}^{-1}$ in the salt solution (virtually identical to that obtained for benzene in the same salt solution). Note that the measured red-shift is independent of pyridine concentration (over a $0.2 m$ to $0.5 m$ range), which implies that the shifts are not due to salt-induced pyridine aggregation, but rather to direct ion-solute interactions. Additionally, the measured frequency shift is insensitive to the specific cation used, as $\Delta v \sim 2 \mathrm{~cm}^{-1}$ in both aqueous NaI and KI solutions. No such salt-induced red-shift was found in similar measurements performed using aqueous KF (in which $\Delta v \sim 0 \pm 0.2 \mathrm{~cm}^{-1}$ ), thus implying that neither $\mathrm{K}^{+}$nor $\mathrm{F}^{-}$have any detectable interaction with either benzene or pyridine (up to a salt concentration of $1 \mathrm{~m}$ ). Previous RamanMCR studies further indicate that $\mathrm{Na}^{+}$does not interact with non-aromatic hydrophobic groups (8). Thus, the above results imply that the aromatic $\mathrm{CH}$ shifts that we have observed in $\mathrm{NaI}$ 
solutions are primarily due to the interaction between $\mathrm{I}^{-}$and benzene (or pyridine).

Fig. $2 \mathrm{~b}$ shows the $\mathrm{I}^{-}$induced $\mathrm{CH}$ shift of both benzene and pyridine as a function of $\mathrm{NaI}$ concentration. These frequency shifts may be used to estimate the corresponding iodide partition coefficient $K_{p}$ by assuming that the observed shifts reflect the probability that a single anion is present in the first hydration shell of the solute, combined with our experimental estimate that one iodide ion in the first hydration-shell induces an aromatic $\mathrm{CH}$ red-shift of $\Delta v_{1}=7 \pm 3 \mathrm{~cm}^{-1}$. This iodide-induced shift was obtained from the frequency of the pyridine $\mathrm{CH}$ peak appearing in the iodide-correlated spectrum (and thus arising from pyridine molecules whose hydration shells contain an iodide ion), as described previously (8). The latter iodide-induced shift is quite similar to the iodide-induced shift in the $\mathrm{CH}$ stretch frequency of non-aromatic methyl groups of $\Delta v_{1}=$ $9 \pm 3 \mathrm{~cm}^{-1}$ (8). Thus, the $K_{p}$ pertaining to the partitioning of iodide ions into the first hydration shell of benzene and pyridine may be estimated as follows:

$$
K_{p}=\frac{1}{N_{H S}} \frac{\left(\Delta v / \Delta v_{1}\right)}{\left(m_{i} / m_{\text {wat }}\right)} \approx 1.54\left(\frac{\Delta v}{\Delta v_{1}}\right)
$$

where $m_{i}$ and $m_{\text {wat }}$ are the molalties of $\mathrm{I}^{-}$and water, respectively, and $N_{H S}=36$ is the MD simulation-derived estimate of the number of water molecules in the first hydration-shell of benzene. The second approximate equality pertains to a $1 \mathrm{~m}$ salt solution.

The primary source of error in the resulting $K_{p}$ values, as reflected in the hatched regions in Fig. 2c, derives from the uncertainty in the shift induced by a single first hydration-shell anion, $\Delta v_{1}$, which is significantly larger than the $\pm 0.5 \mathrm{~cm}^{-1}$ maximum experimental uncertainty in $\Delta v$. Note that the Raman-MCR derived $K_{p}$ values in Fig. 2c imply that both benzene and pyridine have approximately the same $K_{p}$ values. The Raman-MCR derived $K_{p}$ values for $\mathrm{KF}, \mathrm{NaCl}$, and $\mathrm{NaBr}$ were obtained assuming that $\Delta v_{1}=7 \pm 3 \mathrm{~cm}^{-1}$ for all of the anions. Although the latter 
assumption remains to be independently verified, it seems unlikely that the shift induced by a single halogen anion within the first hydration-shell could lie significantly outside this range. However, our preliminary measurements in molecular salt solutions (described below) may imply that these anions induce significantly smaller $\mathrm{CH}$ frequency shifts. The primary finding of our Raman-MCR derived results is that the partition coefficient of $\mathrm{I}^{-}$is somewhat smaller than unity, in agreement with the MD simulation predictions, and significantly smaller than the thermodynamic SPM-based estimate that $K_{p}>1.5$ for $\mathrm{I}^{-}$(Fig. 1b). Moreover, since the $\mathrm{I}^{-}$induced aromatic $\mathrm{CH}$ red-shift of $\sim 2 \mathrm{~cm}^{-1}$ is approximately twice that found previously for non-aromatic $\mathrm{CH}$ groups, our Raman-MCR results imply that $\mathrm{I}^{-}$ions have a higher affinity for the first hydration-shells of aromatic than non-aromatic hydrocarbon groups.

Although our most reproducible Raman-MCR results are those obtained in alkali halide solutions, we have also attempted to use Raman-MCR to quantify the aromatic $\mathrm{CH}$ stretch shift induced by molecular anions in $\mathrm{Na}_{2} \mathrm{SO}_{4}, \mathrm{NaNO}_{3}$, and $\mathrm{NaClO}_{4}$ solutions. Our results indicate that all of these molecular anions produce significantly smaller $\mathrm{CH}$ red-shifts than $\mathrm{I}^{-}$. More specifically, for both benzene and pyridine the $\mathrm{CH}$ red-shifts range from $\Delta v \sim 0.2 \pm 0.3 \mathrm{~cm}^{-1}$ in 1 $m \mathrm{Na}_{2} \mathrm{SO}_{4}$ to $\Delta v \sim 0.5 \pm 0.4 \mathrm{~cm}^{-1}$ in $1 \mathrm{~m} \mathrm{NaClO}_{4}$ (with intermediate values obtained in $1 \mathrm{~m}$ $\mathrm{NaNO}_{3}$ ). The small shift induced by $\mathrm{SO}_{4}{ }^{2-}$ is consistent with the MD results, as well as the assumption of the SPM that $\mathrm{SO}_{4}{ }^{2-}$ is strongly excluded from nonpolar surfaces (20). However, the small red-shift induced by $\mathrm{ClO}_{4}^{-}$is rather surprising, as both the MD and SPM results suggest that this anion should have a substantial affinity for benzene. Thus, our Raman-MCR results may either imply that $\mathrm{ClO}_{4}^{-}$has a quite low value of $K_{p}$ (comparable to that of $\mathrm{Cl}^{-}$) or that the red-shift induced by a single $\mathrm{ClO}_{4}{ }^{-}$anion in the first hydration-shell of benzene (or pyridine) is significantly smaller than that induced by an $\mathrm{I}^{-}$anion. 


\subsection{MD density distributions}

Figs. $3 \mathrm{a}$ and $3 \mathrm{~b}$ show mass density distributions of the cations and anions, respectively, about benzene, obtained from our MD simulations in a cylindrical coordinate system in which the center of the benzene ring is at the origin, and the $z$ axis is normal to the plane of the ring. The anions show significant variability in their density distributions compared to the cations. This is consistent with the observation that cations generally exhibit less pronounced interactions with nonelectrolyte solutes relative to anions (34). The density distributions of potassium and sodium about benzene in $\mathrm{KF}, \mathrm{NaNO}_{3}, \mathrm{NaI}$, and $\mathrm{NaClO}_{4}$ are approximately uniform throughout the hydration layer and bulk water. However, the sodium distributions in the $\mathrm{Na}_{2} \mathrm{SO}_{4}$ and $\mathrm{NaCl}$ systems show some depletion in the vicinity of benzene. This is reflected in the calculated partition coefficients $\left(K_{p}\right)$ for the cations shown in Figure 1a. Of the aqueous solutions studied here, $K_{p}$ for sodium is smallest when in solution with sulfate or chloride. To account for sodium depletion in these two systems, consider the density distributions (Fig. 3a,b) and $K_{p}$ data of sulfate and chloride (Figure 1b). In particular, sulfate is strongly excluded from the hydration layer of benzene, and correspondingly has the smallest $K_{p}$ of all the anions and cations. This result is consistent with other MD studies of air/water and hydrophobic interfaces showing strong exclusion of sulfate $(35,36)$. Furthermore, in $\mathrm{Na}_{2} \mathrm{SO}_{4}$ solution, sodium is observed to be withdrawn by sulfate from the air/water interface (35). Thus, the density depletion about benzene and the low $K_{p}$ values for sodium in $\mathrm{Na}_{2} \mathrm{SO}_{4}$ solution are accounted for by interactions between the ions in solution. Chloride also shows depletion in the benzene hydration layer (Fig. 3b) as well as a relatively small $K_{p}$ value (Fig. 1b). Its exclusion from a hydrophobic surface was also observed in a simulation study of methane in aqueous $\mathrm{NaCl}$ solution (36). Similar to the $\mathrm{Na}_{2} \mathrm{SO}_{4}$ system, a cation-withdrawing mechanism may account for the slight depletion of sodium in the 
hydration layer. According to Collins' "law of similar water affinities" sodium is expected to pair with chloride in aqueous solution (37). A recent study on ion-pairing in sodium salts further corroborates this view (38).

$K_{p}$ data (Fig. 1b) and density distributions (Fig. 3b) indicate exclusion of fluoride from the benzene surface, intermediate between sulfate and chloride. Fluoride exclusion from the air/water $(39,40)$ and macroscopic and molecular hydrophobic interfaces $(8,41,42)$ has been previously demonstrated both in simulations and experimentally. However, potassium, while in the presence of the strong kosmotrope fluoride, shows no appreciable depletion from the benzene surface (Fig. 3b). Ion-pairing would likely not play a role in potassium and fluoride interactions (37). This may account for the unperturbed density distribution of potassium about benzene. However, potassium has been considered a weak chaotrope (37), and hence is expected to show an affinity for the benzene surface. Further studies are needed to elucidate the effect of counteranions on potassium behavior in the presence of nonpolar solutes.

The choatropic anions nitrate, iodide, and perchlorate show an enhanced affinity for the benzene surface relative to the bulk water. Both the density distributions (Fig. 3b) and anion $K_{p}$ values (Fig. 1b) allow for ordering of these anions in decreasing affinity for the benzene surface as $\mathrm{ClO}_{4}^{-}>\mathrm{NO}_{3}{ }^{-}>\mathrm{I}^{-}$. The affinity of perchlorate and iodide for air/water interfaces and hydrophobic surfaces is well established $(13,39,41,43-48)$. While the sodium cation exhibits an overall preference for bulk water, $K_{p}$ values of sodium positively correlate with those of the chaotropic anions (Fig. 1a,b).

Fig. 4 shows normalized density distribution functions, denoted $g(r, z)$, of the anions, cations and water oxygen $\left(\mathrm{O}_{\mathrm{W}}\right)$ atoms around benzene in the $(r, z)$ coordinate system for all six of the simulated salt solutions. The density distributions are normalized by the bulk density of the 
respective species, and they have been symmetrized in the direction $(z)$ normal to the benzene plane. The ion distributions in Fig. 4 are normalized versions of the data shown in Fig. 3. Plotting them along with the $\mathrm{O}_{\mathrm{w}}$ distributions in Fig. 4 enables us to see how the ions are distributed in the benzene hydration shells.

The six different systems show remarkably similar $\mathrm{O}_{\mathrm{W}}$ distributions with an enhanced affinity of water for the benzene surface, especially along the $z$ axis. The solvation of benzene in our simulations is consistent with earlier studies on the hydration of benzene in pure water (49). All of the water distribution functions show a well-defined first hydration shell about benzene peaked at $\sim 3 \AA$ along the $z$ axis and at $\sim 5.5 \AA$ along the radial $(r)$ axis. The structure of the second and third benzene hydration layers depends on the particular anion present in solution. In the presence of the kosmotrope sulfate, there appears to be no water structure beyond the first hydration layer of benzene (Fig. 4a). On the other hand, the choatrope iodide seems to enhance the water structure with a clear indication of a second hydration layer along the $z$ axis, with a peak at $\sim 6 \AA$ above and below the benzene plane; however, along the radial axis there is no clear indication of a second hydration layer (Fig. 4e). The remaining salts show intermediate effects on the hydration characteristics of benzene.

The distributions of the ions depicted in Fig. 4 show markedly different behavior depending on the ions present in the solution. Of the systems simulated, sulfate and sodium in $\mathrm{Na}_{2} \mathrm{SO}_{4}$ show the greatest depletion in the first hydration layer of benzene relative to the other simulated ions (compare Fig. 4a to Fig. 4b-f). Remarkably, there is an enhanced sulfate and sodium density above and below the benzene plane at $|z|>10 \AA$, which is well outside of the first hydration layer. It is not known if this is system size artifact of our simulations or if it implies a long distance interaction between $\mathrm{Na}_{2} \mathrm{SO}_{4}$ and benzene. In the $\mathrm{KF}$ solution, the potassium ion 
exhibits an enhanced affinity for the first hydration layer relative to fluoride, but both ions show an overall depletion relative to the bulk solution (Fig. 4b). Although chloride is excluded from the benzene surface, the distribution function indicates a slight increase in density slightly beyond the first hydration layer of benzene along the $z$ axis.

The chaootropic anions exhibit an enhanced affinity for the hydration layer of benzene. In particular, nitrate and perchlorate density is enhanced at the first minimum of the $\mathrm{O}_{\mathrm{w}}$ density distribution function along the $z$ axis, with perchlorate exhibiting a two-fold enhancement in that region relative to nitrate (Fig. 4d,f). These anion densities are also enhanced along the radial axis coincident with the peak of the first hydration shell of benzene, with perchlorate again exhibiting an almost two-fold enhancement relative to nitrate. Iodide shows enhanced density coincident with the second hydration shell peak at $\sim 6 \AA$ along the $z$ axis (Fig. 4e). However, iodide enhancement along the radial axis resides in the trough beyond the first hydration shell.

\section{Conclusions}

MD simulations of benzene, as well as Raman-MCR measurements of benzene and pyridine, in several salt solutions were performed. Distributions of ions and water around benzene, obtained from $100 \mathrm{~ns}$ simulations, were employed to directly compute ion and salt partition coefficients for comparison with a thermodynamic SPM. The partitioning of the cations into the hydration layer of benzene depended strongly upon the specific counter-anion in solution. The Raman-MCR measurements imply that only anions produce measurable salt induced shifts in the aromatic $\mathrm{CH}$ stretch frequencies of benzene and pyridine. The partition coefficient $K_{p}$ of $\mathrm{I}^{-}$ obtained from these measurements is consistent with that found in the MD simulations, as is the decrease in $K_{p}$ with decreasing size of halogen anions. Good agreement was found between the simulations and the thermodynamic model for the partitioning of salts between the hydration 
layer of benzene and bulk solution. However, inconsistency between this study and the SPM regarding individual cation and anion partitioning suggests that an improved thermodynamic model must account for the interactions between the counter-ions. Greater disparities between the MD simulation results and those obtained using SPM and Raman hydration-shell measurements are found for the partition coefficients for molecular anions. These discrepancies may reflect the influence of molecular polarizability and/or shape on both ion-partitioning and ion-induced aromatic $\mathrm{CH}$ frequency shifts, and possibly also counterion interactions (whose effects were neglected in both the SPM and Raman-MCR analysis).

Our MD simulations have provided an atomic-scale picture of specific-ion interactions with the nonpolar solute benzene. We have shown that nonpolarizable MD simulations are in reasonable (semi-quantitative) agreement with results obtained from both the thermodynamic SPM and Raman-MCR hydration-shell spectroscopic measurements.

\section{Acknowledgements}

The work at UC Irvine was supported by grant CHE-0909227 from the National Science Foundation. The authors at Purdue University acknowledge financial support by grant CHE1213338 from the National Science Foundation. 


\section{Table 1}

Ion force field parameters

\begin{tabular}{ccccc}
\hline Atom & $\sigma[\AA]^{\dagger}$ & $\varepsilon[\mathrm{kcal} / \mathrm{mol}]^{\ddagger}$ & $\mathrm{q}[\mathrm{e}]^{\S}$ & reference \\
\hline $\mathrm{K}^{+}$ & 4.935 & $0.3280 \times 10^{-3}$ & 1.00 & $(50)$ \\
$\mathrm{Na}^{+}$ & 3.330 & $2.771 \times 10^{-3}$ & 1.00 & $(50)$ \\
$\mathrm{F}^{-}$ & 2.733 & 0.7200 & -1.00 & $(51)$ \\
$\mathrm{Cl}^{-}$ & 4.417 & 0.1180 & -1.00 & $(52)$ \\
$\mathrm{I}^{-}$ & 5.400 & 0.0700 & -1.00 & $(51)$ \\
$\mathrm{N}\left(\mathrm{NO}_{3}{ }^{-}\right)$ & 3.900 & 0.8368 & 0.86 & $(53)$ \\
$\mathrm{O}\left(\mathrm{NO}_{3}{ }^{-}\right)$ & 3.154 & 0.6489 & -0.62 & $(53)$ \\
$\mathrm{Cl}\left(\mathrm{ClO}_{4}{ }^{-}\right)$ & 3.950 & 0.9489 & 1.20 & $(54)$ \\
$\mathrm{O}\left(\mathrm{ClO}_{4}^{-}\right)$ & 2.960 & 0.8782 & -0.60 & $(54)$ \\
$\mathrm{S}\left(\mathrm{SO}_{4}{ }^{2-}\right)$ & 3.550 & 0.2500 & 2.00 & $(55)$ \\
$\mathrm{O}\left(\mathrm{SO}_{4}{ }^{2-}\right)$ & 3.150 & 0.2000 & -1.00 & $(55)$ \\
\hline
\end{tabular}

${ }^{\dagger}$ Lennard-Jones size parameter.

Lennard-Jones well depth.

${ }^{\S}$ Atomic charge. 


\section{Figure captions}

Fig. 1. (a) Cation, (b) anion, and (c) total salt partition coefficients, $K_{p}$, calculated from our MD simulations (black bars). The corresponding partition coefficients obtained from a thermodynamic solute partitioning model (SPM) (20) are shown as red bars. In panel (a), the MD values are for $\mathrm{Na}^{+}$in $\mathrm{Na}_{2} \mathrm{SO}_{4}, \mathrm{NaCl}, \mathrm{NaNO}_{3}, \mathrm{NaI}$, and $\mathrm{NaClO}_{4}$, and for $\mathrm{K}^{+}$in $\mathrm{KF}$; the cation values do not depend on the identity of the counter anion in the SPM. In panel (b), the MD values are for $\mathrm{SO}_{4}{ }^{2-}$ in $\mathrm{Na}_{2} \mathrm{SO}_{4}, \mathrm{~F}^{-}$in $\mathrm{KF}, \mathrm{NO}_{3}{ }^{-}$in $\mathrm{NaNO}_{3}, \mathrm{I}^{-}$in $\mathrm{NaI}$, and $\mathrm{ClO}_{4}{ }^{-}$in $\mathrm{NaClO}_{4}$; the anion values do not depend on the identity of the counter cation in the SPM.

Fig. 2. Raman-MCR results for the salt-induced red-shift in aromatic $\mathrm{CH}$ vibrational bands of benzene and pyridine. The solute correlated spectra of $0.5 \mathrm{~m}$ pyridine (solid curves) in both pure water (blue) and $1 \mathrm{~m} \mathrm{NaI}$ (red) are compared with the $\mathrm{OH}$ stretch band of pure water (dashed blue); (a) expanded view of the $\mathrm{CH}$ stretch peak of pyridine, (b) $\mathrm{CH}$ shift of both benzene and pyridine vs. NaI concentration, (c) $K_{p}$ (from Eq. 2) plotted as a function of the salt induced frequency shift of both benzene and pyridine induced by $\mathrm{KF}, \mathrm{NaCl}, \mathrm{NaBr}$, and $\mathrm{NaI}$ (see text for further details).

Fig. 3. Mass density distributions of the (a) cations $\mathrm{Na}^{+}$and $\mathrm{K}^{+}$, and the (b) anions $\mathrm{SO}_{4}{ }^{2-}, \mathrm{F}^{-}, \mathrm{Cl}^{-}$, $\mathrm{NO}_{3}^{-}, \mathrm{I}^{-}$, and $\mathrm{ClO}_{4}^{-}$, around benzene for the six salt systems simulated in this study. The $z$ coordinate runs perpendicular to the benzene plane, with the geometric center of benzene located at the origin. The radial coordinate, $r$, runs radially outward from the $z$-axis parallel to the benzene plane. The distribution at each point $(r, z)$ is an average over the azimuth.

Fig. 4. Normalized density distribution functions of the ions and water oxygen atoms $\left(\mathrm{O}_{\mathrm{W}}\right)$ about benzene. Each distribution is normalized by its bulk density. The $z$ coordinate runs 
perpendicular to the benzene plane, with the geometric center of benzene located at the origin. The radial coordinate, $r$, runs radially outward from the $z$-axis parallel to the benzene plane. The distribution at each point $(r, z)$ is an average over the azimuth. 


\section{REFERENCES}

(1) F Hofmeister: Zur Lehre von der Wirkung der Slaze. Arch. Exp. Pathol. Pharmakol. 24 (1888) 247-60.

(2) Y Zhang, PS Cremer: Chemistry of Hofmeister Anions and Osmolytes. Annu. Rev. Phys. Chem. 61 (2010) 63-83.

(3) KD Collins, MW Washabaugh: The Hofmeister Effect and the Behaviour of Water at Interfaces. Q. Rev. Biophys. 18 (1985) 323-422.

(4) W Kunz, P Lo Nostro, BW Ninhan: The Present State of Affairs with Hofmeister Effects. Curr. Opin. Coll. Int. Sci. 9 (2004) 1-18.

(5) Y Marcus: Effect of Ions on the Structure of Water: Structure Making and Breaking. Chem. Rev. 109 (2009) 1346-70.

(6) JD Smith, RJ Saykally, PL Geissler: The Effects of Dissolved Halide Anions on Hydrogen Bonding in Liquid Water. J. Am. Chem. Soc. 129 (2007) 13847-56.

(7) PN Perera, B Browder, D Ben-Amotz: Perturbations of Water by Alkali Halide Ions Measured using Multivariate Raman Curve Resolution. J. Phys. Chem. B 113 (2009) 180509.

(8) BM Rankin, D Ben-Amotz: Expulsion of Ions from Hydrophobic Hydration Shells. J. Am. Chem. Soc. 135 (2013) 8818-21.

(9) AW Omta, MF Kropman, S Woutersen, HJ Bakker: Negligible Effect of Ions on Hydrogen-Bond Structure in Liquid Water. Science (2003).

(10) CD Cappa, JD Smith, KR Wilson, BM Messer, MK Gilles, RC Cohen, RJ Saykally: Effects of Alkali Metal Halide Salts on the Hydrogen Bond Network of Liquid Water. J. Phys. Chem. B 109 (2005) 7046-52.

(11) DA Schmidt, O Birer, S Funkner, BP Born, R Gnanasekaran, G Schwaab, DM Leitner, M Havenith: Rattling in the Cage: Ions as Probes of Sub-picosecond Water Network Dynamics. J. Am. Chem. Soc. 131 (2009) 18512-17.

(12) S Funkner, G Niehues, DA Schmidt, M Heyden, G Schwaab, KM Callahan, DJ Tobias, M Havenith: Watching the Low-Frequency Motions in Aqueous Salt Solutions: The Terahertz Vibrational Signatures of Hydrated Ions. J. Am. Chem. Soc. 134 (2012) 1030-35.

(13) P Jungwirth, DJ Tobias: Specific Ion Effects at the Air/Water Interface. Chem. Rev. 106 (2006) 1259-81.

(14) P Lo Nostro, BW Ninham: Hofmeister Phenomena: An Update on Ion Specificity in Biology. Chem. Rev. 112 (2012) 2286-322.

(15) P Jungwirth, PS Cremer: Beyond Hofmeister. Nat. Chem. 6 (2014) 261-63.

(16) DJ Tobias, JC Hemminger: Chemistry: Getting Specific About Specific Ion Effects. Science 319 (2008) 1197-98.

(17) DE Otten, PR Shaffer, PL Geissler, RJ Saykally: Elucidating the Mechanism of Selective Ion Adsorption to the Liquid Water Surface. Proc. Natl. Acad. Sci. USA 109 (2012) 70105.

(18) A Arslanargin, TL Beck: Free Energy Partitioning Analysis of the Driving Forces that Determine Ion Density Profiles Near the Water Liquid-Vapor Interface. J. Chem. Phys. 136 (2012) 104503.

(19) DJ Tobias, AC Stern, MD Baer, Y Levin, CJ Mundy: Simulation and Theory of Ions at Atmosperically Relevant Aqueous Liquid-Air Interfaces. Annu. Rev. Phys. Chem. 64 (2013) 339-59.

(20) LM Pegram, MT Record Jr.: Thermodynamic Origin of Hofmeister Ion Effects. J. Phys. Chem. B 112 (2008) 9428-36.

(21) LM Pegram, MT Record Jr.: Partitioning of Atmospherically Relevant Ions between Bulk Water and the Water/Vapor Interface. Proc. Natl. Acad. Sci. USA 103 (2006) 14278-81.

(22) LM Pegram, MT Record Jr.: Hofmeister Salt Effects on Surface Tension Arise from Partitioning of Anions and Cations between Bulk Water and the Air-Water Interface. J. Phys. Chem. B 111 (2007) 5411-17. 
(23) WL Jorgensen, J Chandrasekhar, JD Madura, RW Impey, ML Klein: Comparison of Simple Potential Functions for Simulating Liquid Water. J. Chem. Phys. 79 (1983) 926-35.

(24) WL Jorgensen, DL Severance: Aromatic-Aromatic Interactions: Free Energy Profiles for the Benzene Dimer in Water, Chloroform, and Liquid Benzene. J. Am. Chem. Soc. 112 (1990) 4768-74.

(25) U Essmann, L Perera, ML Berkowitz, T Darden, LG Pedersen: A Smooth Particle Mesh Ewald Method. J. Chem. Phys. 103 (1995) 8577-93.

(26) HJC Berendsen, JPM Postma, WF van Gunsteren, A DiNola, JR Haak: MolecularDynamics with Coupling to an External Bath. J. Chem. Phys. 81 (1984) 3684-90.

(27) J-P Ryckaert, G Ciccotti, HJC Berendsen: Numerical Integration of the Cartesian Equations of Motion of a System with Constraints: Molecular Dynamics of $n$-Alkanes. J. Comp. Phys. 23 (1977) 327-41.

(28) DA Case, TA Darden, TE Cheatham III, CL Simmerling, J Wang, RE Duke, R Luo, KM Merz, B Wang, DA Pearlman, M Crowley, S Brozell, V Tsui, H Gohlke, J Mongan, V Hornak, G Cui, P Beroza, C Schafmeister, JW Caldwell, WS Ross, PA Kollman: AMBER 8. (2004) University of California, San Francisco.

(29) W Humphrey, W Dalke, K Schulten: VMD: Visual Molecular Dynamics. J. Mol. Graph. 14 (1996) 33-38.

(30) JG Davis, KP Gierszal, P Wang, D Ben-Amotz: Water Structural Transformation at Molecular Hydrophobic Interfaces. Nature 491 (2012) 582-85.

(31) WH Lawton, EA Sylvestre: Self Modeling Curve Resolution. Technometrics 13 (1971) 617-33.

(32) J Heyda, JC Vincent, DJ Tobias, J Dzubiella, P Jungwirth: Ion Specificity at the Peptide Bond: Molecular Dynamics Simulations of N-Methylacetamide in Aqueous Salt Solutions. J. Phys. Chem. B 114 (2010) 1213-20.

(33) KP Gierszal, JG Davis, MD Hands, DS Wilcox, LV Slipchenko, D Ben-Amotz: $\pi$ Hydrogen Bonding in Liquid Water. J. Phys. Chem. Lett. 2 (2011) 2930-33.

(34) MG Cacace, EM Landau, JJ Ramsden: The Hofmeister Series: Salt and Solvent Effects on Interfacial Phenomena. Q. Rev. Biophys. 30 (1997) 241-77.

(35) S Gopalakrishnan, P Jungwirth, DJ Tobias, HC Allen: Air-Liquid Interfaces of Aqueous Solutions Containing Ammonium and Sulfate: Spectroscopic and Molecular Dynamics Studies. J. Phys. Chem. B 109 (2005) 8861-72.

(36) PE Smith: Computer Simulation of Cosolvent Effects on Hydrophobic Hydration. J. Phys. Chem. B 103 (1999) 525-34.

(37) KD Collins: Ions from the Hofmeister Series and Osmolytes: Effects on Proteins in Solution and in the Crystallization Process. Methods 34 (2004) 300-11.

(38) B Jagoda-Cwiklik, R Vácha, M Lund, M Srebro, P Jungwirth: Ion Pairing as a Possible Clue for Discriminating between Sodium and Potassium in Biological and Other Complex Environments. J. Phys. Chem. B 111 (2007) 14077-79.

(39) P Jungwirth, DJ Tobias: Molecular Structure of Salt Solutions: A New View of the Interface with Implications for Heterogeneous Atmospheric Chemistry. J. Phys. Chem. B 105 (2001) 10468-72.

(40) MA Brown, RD D'Auria, I-FW Kuo, MJ Krisch, DE Starr, H Bluhm, DJ Tobias, JC Hemminger: Ion Spatial Distributions at the Liquid-Vapor Interface of Aqueous Potassium Fluoride Solutions. Phys. Chem. Chem. Phys. 10 (2008) 4778-84.

(41) S Pal, F Müller-Plathe: Molecular Dynamics Simulation of Aqueous NaF and NaI Solutions near a Hydrophobic Surface. J. Phys. Chem. B 109 (2005) 6405-15.

(42) BM Rankin, MD Hands, DS Wilcox, KR Fega, LV Slipchenko, D Ben-Amotz: Interactions between Halide Anions and a Molecular Hydrophobic Interface. Faraday Discuss. 160 (2013) 255-70.

(43) P Jungwirth, DJ Tobias: Ions at the Air/Water Interface. J. Phys. Chem. B 106 (2002) 6361-73.

(44) S Ghosal, JC Hemminger, H Bluhm, BS Mun, ELD Hebenstreit, G Ketteler, DF Ogletree, FG Requejo, M Salmeron: Electron Spectroscopy of Aqueous Solution Interfaces Reveals 
Surface Enhancement of Halides. Science 307 (2005) 563-66.

(45) D Horinek, RR Netz: Specific Ion Adsorption at Hyrdophobic Solid Surfaces. Phys. Rev. Lett. 99 (2007) 226104.

(46) W Hua, D Verreault, HC Allen: Surface Prevalence of Perchlorate Anions at the Air/Aqueous Interface. J. Phys. Chem. Lett. 4 (2013) 4231-36.

(47) MD Baer, I-FW Kuo, H Bluhm, S Ghosal: Behavior of Perchlorate versus Chloride Ions in Aqueous Solutions. J. Phys. Chem. B 113 (2009) 15843-50.

(48) J Cheng, CD Vecitis, MR Hoffmann, AJ Colussi: Experimental Anion Affinities for the Air/Water Interface. J. Phys. Chem. B 110 (2006) 25598-602.

(49) TM Raschke, M Levitt: Detailed Hydration Maps of Benzene and Cyclohexane Reveall Distinct Water Structures. J. Phys. Chem. B 108 (2004) 13492-500.

(50) J Aqvist: Ion-Water Interaction Potentials Derived from Free Energy Perturbation Simulations. J. Phys. Chem. 94 (1990) 8021-24.

(51) NA McDonald, EM Duffy, WL Jorgensen: Monte Carlo Investigations of Selective Anion Complexation by a Bis(phenylurea) p-tert-Butylcalix[4]arene. J. Am. Chem. Soc. 120 (1998) 5104-11.

(52) J Chandrasekhar, DC Spellmeyer, WL Jorgensen: Energy Component Analysis for Dilute Aqueous Solutions of Li+, Na+, F-, and Cl- Ions. J. Am. Chem. Soc. 106 (1984) 903-10.

(53) T Megyes, S Balint, E Peter, T Grosz, I Bako, H Krienke, MC Bellissent-Funel: Solution Structure of NaNO3 in Water: Diffraction and Molecular Dynamics Simulation Study. J. Phys. Chem. B 113 (2009) 4054-64.

(54) T Li, PB Balbuena: Theoretical Studies of Lithium Perchlorate in Ethylene Carbonate, Propylene Carbonate, and their Mixtures. J. Electrochem. Soc. 146 (1999) 3613-22.

(55) WR Cannon, BM Pettitt, JA McCammon: Sulfate Anion in Water: Model Structural, Thermodynamic, and Dynamic Properties. J. Phys. Chem. 98 (1994) 6225-30. 
$K_{p \text {,cation }}$

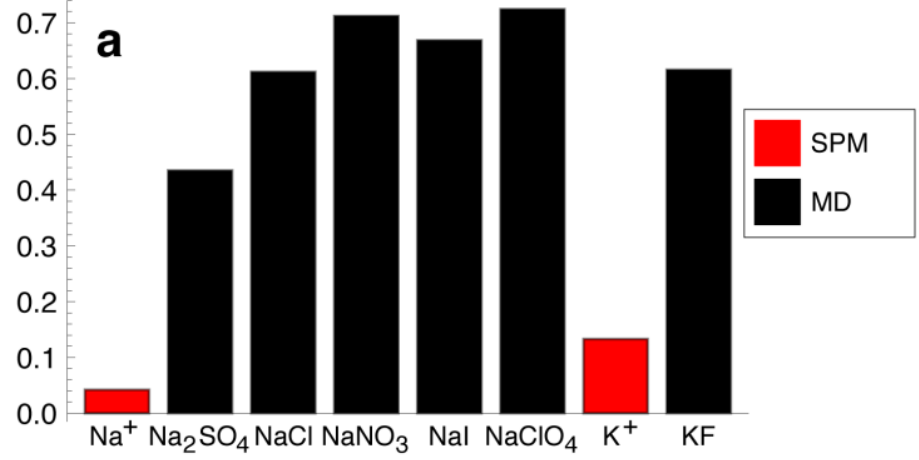

$K_{p \text {,anion }}$
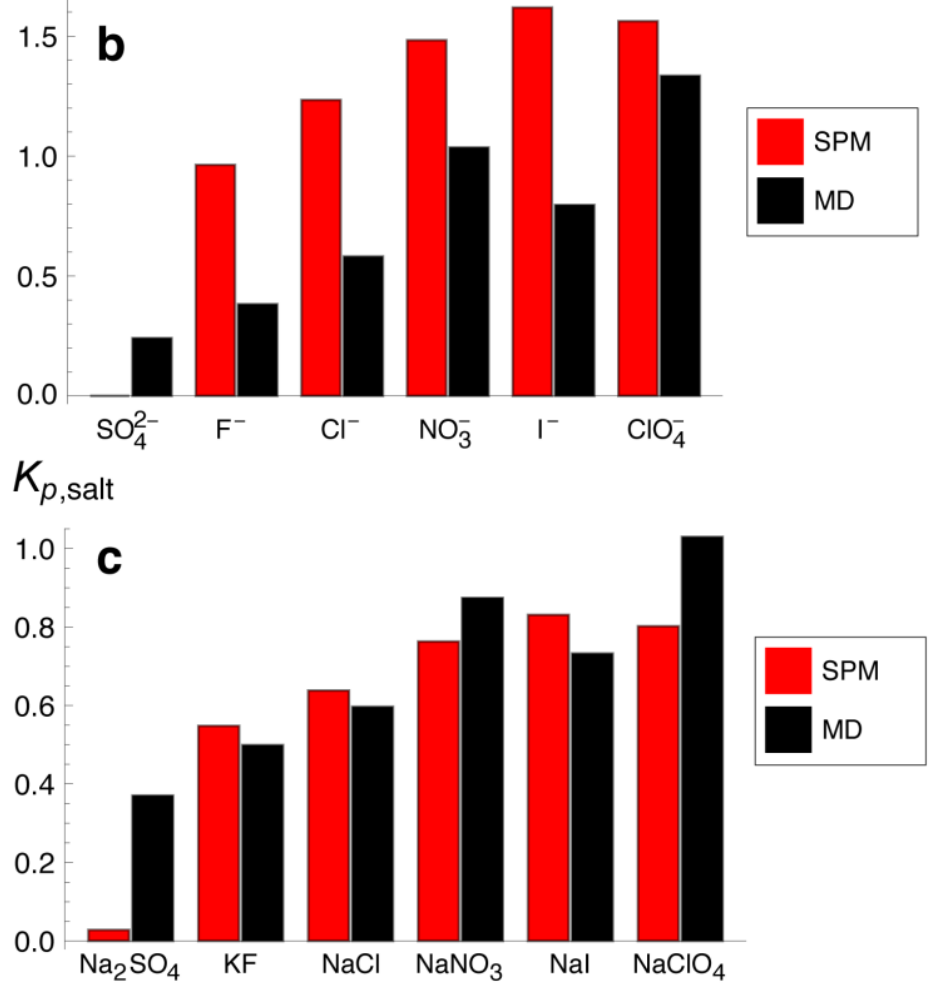

Figure 1 


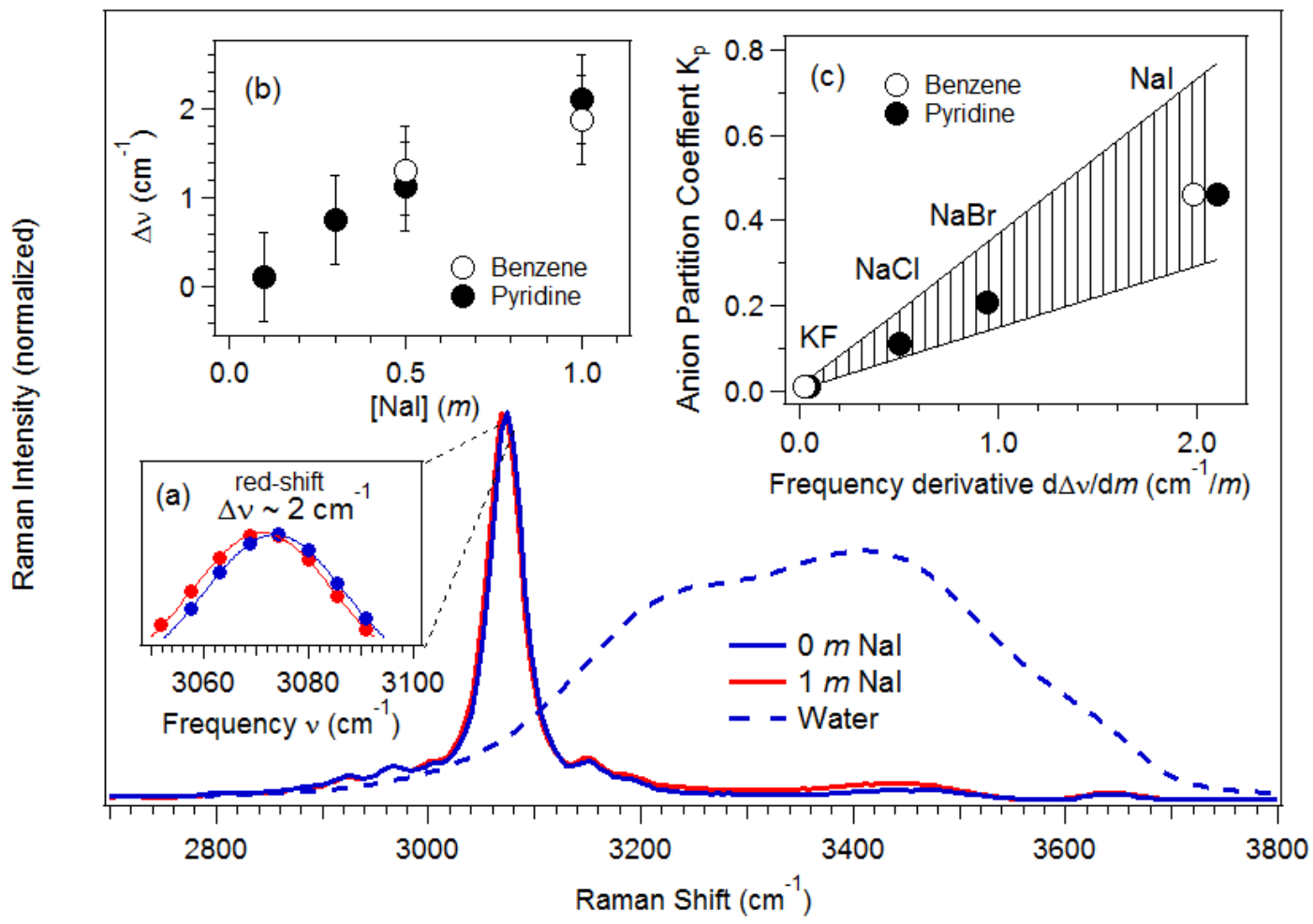

Figure 2 


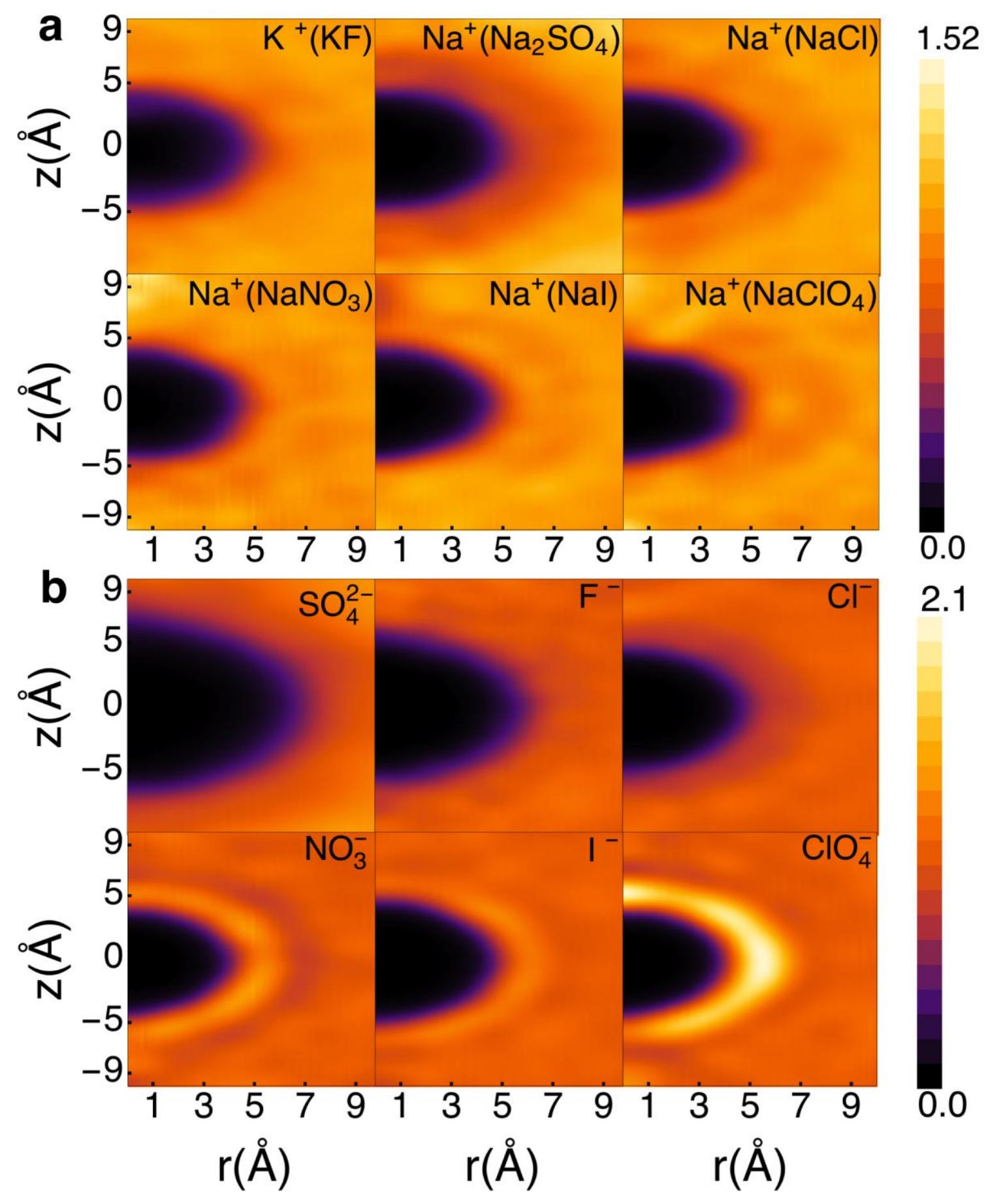

Figure 3 

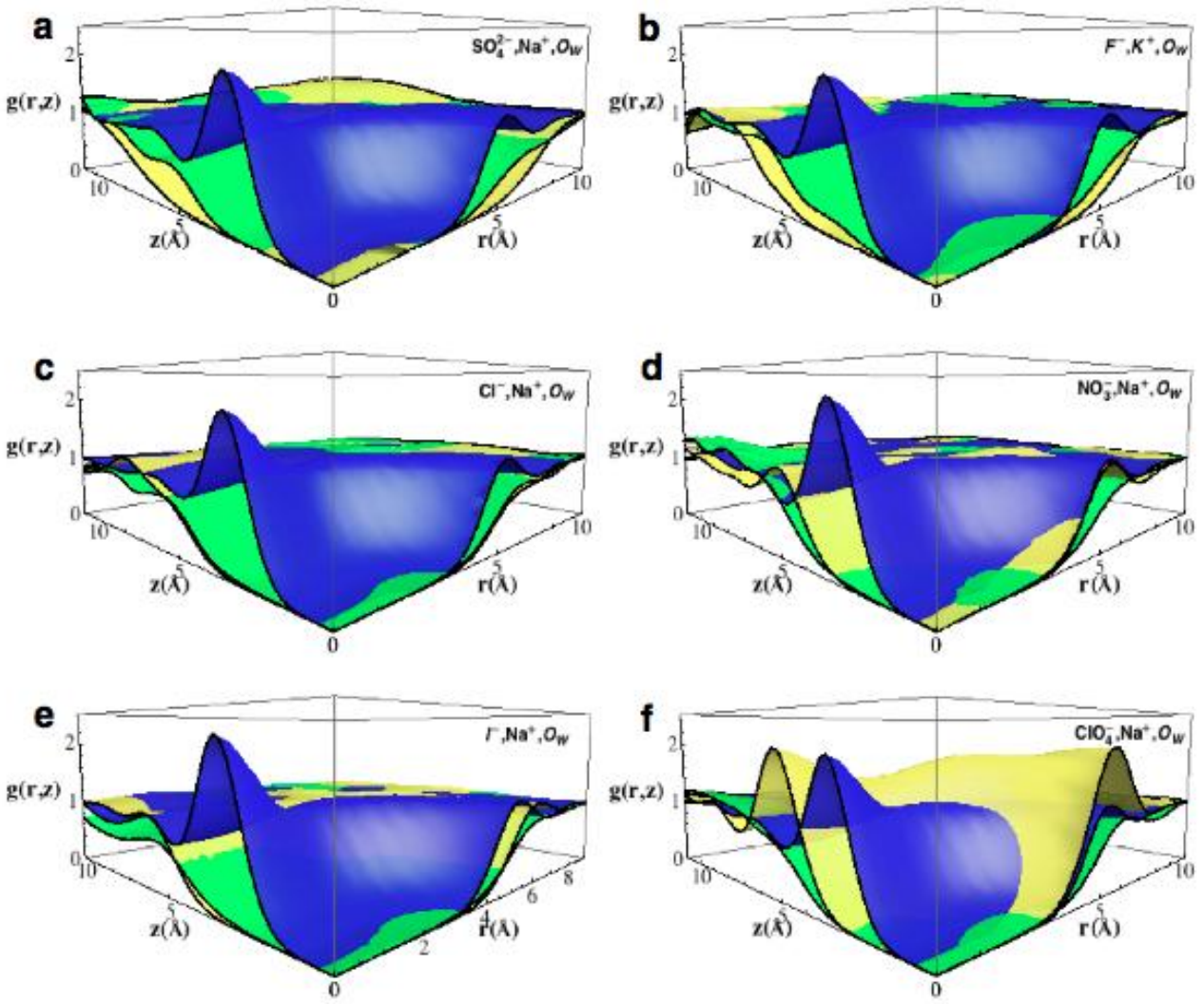

Figure 4 
Graphical Abstract

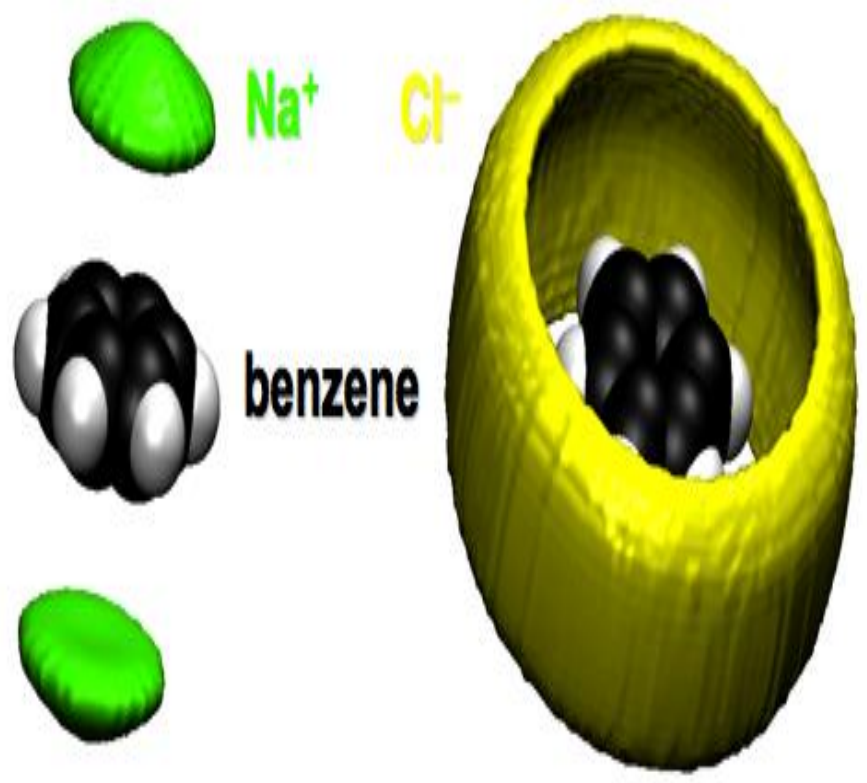


Jordan Vincent received his B. S. in Chemistry and B. A. in Mathematics from Saint Cloud State University in 2007, and an M. S. in Chemistry from the UC Irvine, in 2011. He is currently a $\mathrm{Ph}$. D. candidate at UC Irvine. His research interests include development and application of $\mathrm{ab}$ initio non-adiabatic and classical molecular simulation techniques to aqueous interface chemistry problems and modeling photochemical reaction dynamics.

Sarah M. Matt obtained her B.A. degree in chemistry from Wartburg College in 2012. She is currently pursuing her Ph. D. in chemistry at Purdue University and is working with Dor BenAmotz. Her current research interests include Raman hydration-shell spectroscopy of aqueous systems and multivariate hyperspectral imaging.

Blake M. Rankin obtained his B. S. and B. A. degrees in Accounting and Chemistry, respectively, from the University of Tampa in 2011 and his Ph. D. in Chemistry from Purdue University in 2015. He was awarded a National Research Council Research Associateship at Wright Patterson Air Force Base in 2015 to conduct hyperspectral imaging research for target detection applications.

Raffaella D'Auria obtained a Laurea in Physics from the University of Rome, "La Sapienza" in 1997 and a Ph. D. in Atmospheric Sciences from UCLA in 2005. Her Ph.D. work concentrated on atmospheric aerosols nucleation using ab initio simulations. From 2005 to 2008 she held a postdoctoral position at UC Irvine where she worked on aqueous interfaces by means of classical and ab initio molecular dynamics. In 2009 she joined the Institute of Digital Research and Education at UCLA where she has been working in high performance computing. She is also a lecturer for the Institute of the Environment at UCLA.

J. Alfredo Freites earned his Ph. D. in physics at UC Irvine in 2004. He is currently a project scientist in the department of chemistry at UC Irvine. He studies structure, dynamics and function of proteins, membranes and soft interfaces using theoretical and computational methods.

Dor Ben-Amotz obtained his B. S. degree from Bennington College in 1976 and his Ph.D. from U. C. Berkeley in 1986, followed by a postdoctoral fellowship with Dudley Herschbach at the Exxon Corporate Research Lab in Annandale, NJ. He has been a faculty member in the Department of Chemistry at Purdue University since 1989, where he received an NSF Presidential Young Investigator Award in 1991, and Charles B. Murphy Outstanding Undergraduate Teaching Award in 2012. His recent experimental and theoretical interests include hydration-shell spectroscopy, liquid theory, hyperspectral compressive imaging, and new ways of teaching physical chemistry.

Douglas J. Tobias obtained his B. S. and M.S. in Chemistry at UC Riverside, in 1984 and 1985, respectively, and his $\mathrm{Ph}$. D. in Chemistry/Biophysics at Carnegie Mellon University in 1991. He was a postdoctoral researcher at the University of Pennsylvania from 1991-1995, and a guest researcher at the NIST Center for Neutron Research from 1995-1997. He was appointed to the faculty in the department of chemistry at UC Irvine in 1997. He is a fellow of the American Association for the Advancement of Science, American Chemical Society, and American Physical Society. His research interests include the development and application of molecular 
simulation techniques to the study of membrane biophysics, atmospheric chemistry, and aqueous interfaces. 
Jordan Vincent:

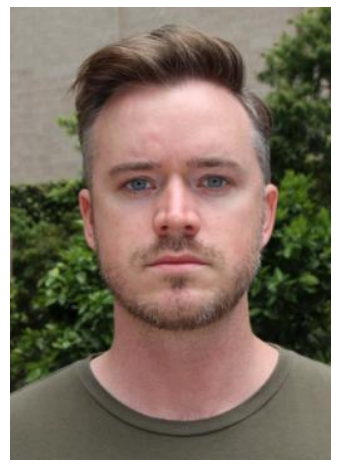

Sarah M. Matt:

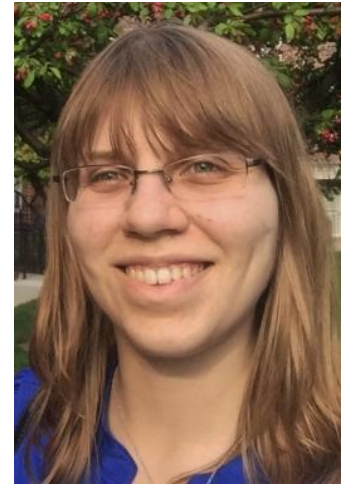

Blake M. Rankin:

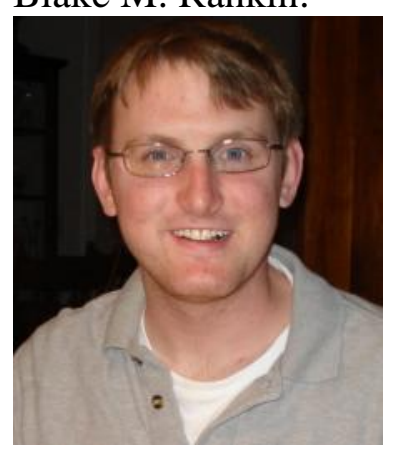

Raffaella D'Auria:

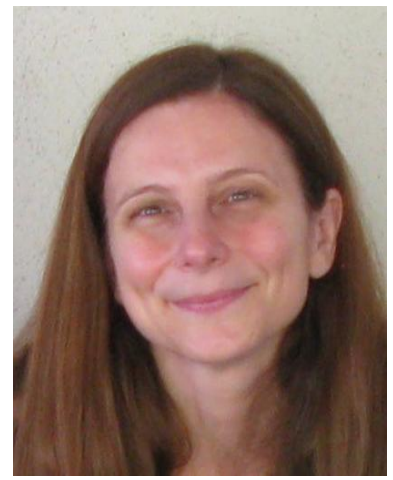




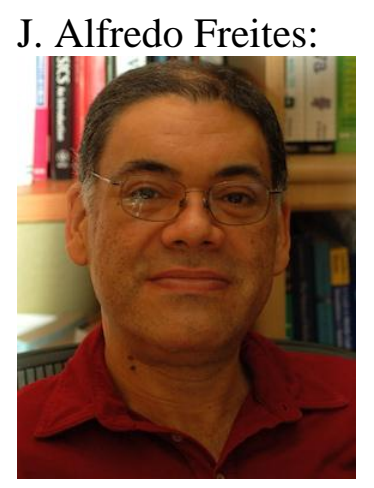

Dor Ben-Amotz:

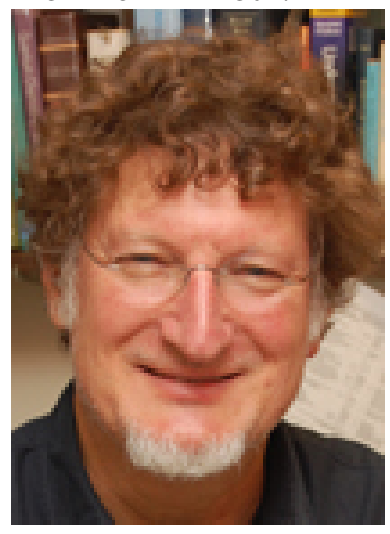

Douglas J. Tobias:

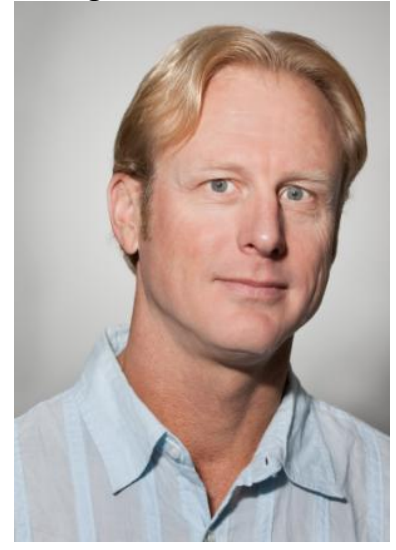

